\title{
Can power corrections be reliably computed in models with extra dimensions?
}

\author{
J.F. Oliver, J. Papavassiliou, and A. Santamaria \\ Departament de Fúsica Tè̀rica and IFIC, Universitat de València-CSIC \\ Dr. Moliner 50, E-46100 Burjassot (València), Spain
}

\begin{abstract}
We critically revisit the issue of power-law running in models with extra dimensions. The analysis is carried out in the context of a higher-dimensional extension of QED, with the extra dimensions compactified on a torus. It is shown that a naive $\beta$ function, which simply counts the number of modes, depends crucially on the way the thresholds of the Kaluza-Klein modes are crossed. To solve these ambiguities we turn to the vacuum polarization, which, due to its special unitarity properties, guarantees the physical decoupling of the heavy modes. This latter quantity, calculated in the context of dimensional regularization, is used for connecting the low energy gauge coupling with the coupling of the $D$-dimensional effective field theory. We find that the resulting relation contains only logarithms of the relevant scales, and no power corrections. If, instead, hard cutoffs are used to regularize the theory, one finds power corrections, which could be interpreted as an additional matching between the effective higher-dimensional model and some unknown, more complete theory. The possibility of estimating this matching is examined in the context of a toy model. The general conclusion is that, in the absence of any additional physical principle, the power corrections depend strongly on the details of the underlying theory. Possible consequences of this analysis for gauge coupling unification in theories with extra dimensions are briefly discussed.
\end{abstract}

PACS numbers: 11.10.Kk,11.10.Hi,12.10.Dm,12.10.Kt 


\section{INTRODUCTION}

The study of models with extra dimensions has received a great deal of attention recently [1, 2, 3, 4], mainly because of the plethora of theoretical and phenomenological ideas associated with them, and the flexibility they offer for realizing new, previously impossible, field-theoretic constructions. One of the most characteristic features of such models is that of the "early unification": the running of gauge couplings is supposed to be modified so strongly by the presence of the tower of KK modes, that instead of logarithmic it becomes linear, quadratic, etc, depending on the number of extra dimensions [5, 6, 7, 8, 9, 10, 11, 12, 13, 14, 15, 16, 17, 18, 19, 20, 21]. Specifically, it has been widely argued that the gauge couplings run as $\mu^{\delta}$, where the $\delta$ is the number of compact extra dimensions. Thus, if the extra dimensions are sufficiently large, such a behavior of the couplings could allow for their unification at accessible energies, of the order of a few $\mathrm{TeV}$, clearly an exciting possibility.

The assertion that gauge-couplings display power-law running is based on rather intuitive arguments: In $\overline{\mathrm{MS}}$ schemes the QED $\beta$ function is proportional to the number of "active" flavors, namely the number of particles lighter than the renormalization scale. Using this argument, and just counting the number of modes lighter than $\mu$, one easily finds that the " $\beta$ function" of QED in models with extra dimensions grows as $\mu^{\delta}$. This behavior is also justified by explicit calculations of the vacuum polarization of the photon using hard cutoffs; since the cutoff cannot be removed, due to the non-renormalizability of the theory, it is finally identified with the renormalization scale, a procedure which eventually leads to a similar conclusion [6, 7] (but with the final coefficient adjusted by hand in order to match the naive expectation in $\overline{\mathrm{MS}})$.

Even though these arguments are plausible, the importance of their consequences requires that they should be scrutinized more carefully [22]. In particular, the argument based on $\overline{\mathrm{MS}}$ running is rather tricky. As it is well known, the $\overline{\mathrm{MS}}$ scheme, because of its mass independence, does not satisfy decoupling, already at the level of four-dimensional theories. Instead, decoupling has to be imposed by hand every time a threshold is passed: one builds an effective theory below the threshold, $m$, and matches it to the theory above the threshold. This matching is carried out by requiring that some physical amplitude or Green's function (i.e. the effective charge) is the same when calculated using either theory, at energies where 
both theories are reliable, namely at $Q^{2}$ much below the threshold. Then, since the renormalization scale, $\mu$, is still a free parameter, one chooses $\mu$ around $m$, in order to avoid large logarithms in the matching equations. In the case of gauge couplings and $\overline{\mathrm{MS}}$ schemes with $\operatorname{Tr}\left\{I_{\text {Dirac }}\right\}=4$ one finds (at one loop) that gauge couplings are continuous at $\mu=m$. This statement is, however, extremely scheme dependent: just by choosing $\operatorname{Tr}\left\{I_{\text {Dirac }}\right\}=2^{D / 2}$ it gets completely modified (see for instance [23]) . In addition to these standard ambiguities, a new complication arises in the context of higher-dimensional models. In particular, the aforementioned procedure requires that the different scales be widely separated in order to avoid that higher dimension operators, generated in the process of matching, become important. However, the condition of having well-separated thresholds is rather marginally satisfied in the case of an infinite tower of KK modes with $M_{n}=n M_{c}\left(M_{c}\right.$ is the compactification scale). In fact, as we will see in detail later, the results obtained for a $\beta$ function that just counts the number of active modes depend very strongly on the prescription chosen for the way the various thresholds are crossed.

As has been hinted above, the deeper reason behind these additional type of ambiguities is the fact that, gauge theories in more than 4 dimensions, compactified or not, are not renormalizable. At the level of the 4-dimensional theory with an infinite number of KK modes the non-renormalizability manifests itself by the appearance of extra divergences, encountered when summing over all the modes. If the theory is not compactified the non-renormalizability is even more evident, since gauge couplings in theories with $\delta$ extra dimensions have dimension $1 / M^{\delta / 2}$. Therefore, gauge theories in extra dimensions should be treated as effective field theories (EFT). Working with such theories presents several difficulties, but, as we have learned in recent years, they can also be very useful. In the case of quantum field theories in extra dimensions, there is no alternative: basic questions, such as the calculation of observables or the unification of couplings, can only be addressed in the framework of the EFT's. However, before attempting to answer specific questions related to the running of couplings in the extra-dimensional theories, one should first clarify the type of EFT one is going to use, since there are, at least, two types of EFT [24]: In one type, known as "Wilsonian EFT" (WEFT) [25], one keeps only momenta below some scale $\Lambda$, while all the effects of higher momenta or heavy particles are encoded in the couplings of the effective theory. This method is very intuitive and leads, by definition, to finite results at each step; however, the presence of the cutoff in all expressions makes the method cumbersome to use, and in the particular 
case of gauge theories difficult to reconcile with gauge-invariance. The WEFT approach has already been applied to the problem of running of couplings in theories with compact extra dimensions, but only for the case of scalar theories [21]. Within the context of another type of EFT, often termed "continuum effective field theories" (CEFT) (see for instance $[24,26,27,28,29,30,31])$, one allows the momenta of particles to vary up to infinity, but heavy particles are removed from the spectrum at low energies. As in the WEFT case the effects of heavier particles are absorbed into the coefficients of higher dimension operators. Since the momenta are allowed to be infinite, divergences appear, and therefore the CEFT need to undergo both regularization and renormalization. In choosing the specific scheme for carrying out the above procedures particular care is needed. Whereas in principle one could use any scheme, experience has shown that the most natural scheme for studying the CEFT is dimensional regularization with minimal subtraction [24, 26, 27, 28, 29, 30, 31]. CEFT are widely used in Physics: for example, when in the context of QCD one talks about 3, 4 or 5 active flavors, one is implicitly using this latter type of effective theories [27, 32]. Moreover, most of the analyses of Grand Unification [28, 33] resort to CEFT-type of constructions: one has a full theory at the GUT scale, then an effective field theory below the GUT scale (SM or MSSM) is built, and then yet another effective field theory below the Fermi scale (just $\mathrm{QED}+\mathrm{QCD})$. In these cases the complete theory is known, and the CEFT language is used only in order to simplify the calculations at low energies and to control the large logarithms which appear when there are widely separated scales. Nevertheless, CEFT's are useful even when the complete theory is not known, or when the connection with the complete theory cannot be worked out; this is the case of Chiral Perturbation theory $(\chi P T)$ [34, 35, 36, 37] (for more recent reviews see also [29, 38, 39]).

It is important to maintain a sharp distinction between the two types of EFT mentioned above, i.e. Wilsonian or continuum, because conceptually they are quite different. However, perhaps due to the fact that the language is in part common to both types of theories, it seems that they are often used interchangeably in the literature, especially when employing cutoffs within the CEFT framework. In particular, since the couplings $\alpha_{i}$ have dimensions $\left[\alpha_{i}\right]=M^{-n}$, when computing loops one generally obtains effects which grow as $\left(\Lambda^{n} \alpha_{i}\right)^{m}$, where $\Lambda$ is the formal CEFT cutoff, and as such is void of physics. As a consequence, physical observables should be made as independent of these cutoffs as possible by introducing as many counterterms as needed to renormalize the answer. Not performing these renor- 
malizations correctly, or identifying naively formal cutoffs with the physical cutoffs of the effective theory, can lead to completely non-sensical results (see for instance [40, 41]). This type of pitfalls may be avoided by simply using dimensional regularization, since the latter has the special property of not mixing operators with different dimensionalites.

The usual way to treat theories with compactified extra dimensions is to define them as a 4-dimensional theory with a truncated tower of KK modes at some large but otherwise arbitrary $N_{s}$, a procedure which effectively amounts to using a hard cutoff in the momenta of the extra dimensions. Thus, physical quantities calculated in this scheme depend explicitly on the cutoff $N_{s}$, which is subsequently identified with some physical cutoff. However, as already commented, $N_{s}$ plays the role of a formal cutoff, and is therefore plagued with all the aforementioned ambiguities. Identification of this formal cutoff with a universal physical cutoff can give the illusion of predictability, making us forget that we are dealing with a non-renormalizable theory with infinite number of parameters, which can be predictive only at low energies, where higher dimension operators may be neglected.

In this paper we want to analyze the question of the running of gauge couplings in theories with compact dimensions from the CEFT "canonical" point of view. We hasten to emphasize that even the CEFT presents conceptual problems in theories with compactified dimensions. Specifically, as mentioned above, in the CEFT approach the (virtual) momenta are allowed to vary up to infinity; however, momenta related to the compactified extra dimensions turn out to be KK masses in the 4-dimensional compactified theory, where it is supposed that one only keeps particles lighter than the relevant scale. Thus, truncating the KK series amounts to cutting off the momenta of the compactified dimensions. Therefore, in order to define a true "non-cutoff" CEFT scheme we are forced to keep all KK modes. Our main motivation is to seriously explore this approach, and investigate both its virtues and its limitations for the problem at hand. We hope that this study will help us identify more clearly which quantities can and which cannot be computed in effective extra-dimensional theories.

The paper is organized as follows: In section @ we discuss the usual arguments in favor of power-law running of gauge couplings and show that they depend crucially on the way KK thresholds are crossed. In particular we show that, a one-loop $\beta$ function which simply counts the number of modes, diverges for more than 5 dimensions, if the physical way of passing thresholds dictated by the vacuum polarization function (VPF) is imposed.

In section III we introduce a theory with one fermion and one photon in $4+\delta$ dimensions, 
with the extra $\delta$ ones compactified. This theory, which is essentially QED in $4+\delta$ dimensions, serves as toy model for studying the issue of power corrections and the running of the coupling in a definite framework.

In section IV we study the question of decoupling KK modes in the aforementioned theory by analyzing the behavior of the VPF of the (zero-mode) photon. Since, as commented above, decoupling the KK modes one by one is problematic, we study the question of how to decouple all of them at once. To accomplish this we consider the VPF of the photon with all KK modes included, and study how it reduces at $Q^{2} \ll M_{c}$ to the standard QED VPF with only one light mode. Since the entire KK tower is kept untruncated, the theory is of course non-renormalizable; therefore, to compute the VPF we have to regularize and renormalize it in the spirit of the CEFT, in a similar way that observables are defined in $\chi P T$. As in $\chi P T$, it is most convenient to use dimensional regularization with minimal subtraction, in order to maintain a better control on the mixing among different operators. However, at the level of the 4-dimensional theory the non-renormalizability manifest itself through the appearance of divergent sums over the infinite KK modes, and dimensional regularization does no seem to help in regularizing them. The dimensional regularization of the VPF is eventually accomplished by exploiting the fact that its UV behavior coincides to that found when the $\delta$ extra dimensions have not been compactified ${ }^{1}$. To explore this point we first resort to the standard unitarity relation (optical theorem), which relates the imaginary part of the VPF to the total cross section in the presence of the KK modes; the latter is finite because the phase-space truncates the series. For $Q^{2} \gg M_{c}^{2}$ the uncompactified result for the imaginary part of the VPF is rapidly reached, i.e. after passing a few thresholds. We then compute the real part of the one-loop VPF in the non-compact theory in $4+\delta$ dimensions, where, of course we can use directly dimensional regularization to regularize it (since no KK reduction has taken place). For later use we also present results in which the same quantity is evaluated by using hard cutoffs. Finally, we show that the UV divergences of the one-loop VPF are indeed the same in both the (torus)-compactified and uncompactified theories. Therefore, in order to regularize the VPF in the compactified theory with an infinite number of KK modes it is sufficient to split the VPF into two pieces, an "uncompactified"

\footnotetext{
${ }^{1}$ This is in a way expected, since for very large $Q^{2} \gg M_{c}=1 / R_{c}$ the compactification effects should be negligible. Note, however, that this is not always the case; a known exception is provided by the orbifold compactification [22].
} 
piece, corresponding to the case where the extra dimensions are treated at the same footing as the four usual ones, and a piece which contains all compactification effects. We show that this latter piece is UV and IR finite and proceed to evaluate it, while all UV divergences remain in the former, which we evaluate using dimensional regularization.

The results of previous sections are used in section $\nabla$ to define an effective charge $\alpha_{\text {eff }}(Q)$ which can be continuously extrapolated from $Q^{2} \ll M_{c}$ to $Q^{2} \gg M_{c}$. We use this effective charge to study the matching of couplings in the low energy effective theory (QED) to the couplings of the theory containing an infinite of KK modes. In the context of dimensional regularization we find that this matching contains only the standard logarithmic running from $m_{Z}$ to the compactification scale $M_{c}$, with no power corrections. On the other hand, if hard cutoffs are used to regularize the VPF in the non-compact space, one does find power corrections, which may be interpreted as an additional matching between the effective $D=4+\delta$ dimensional field theory and some more complete theory. We discuss the possibility of estimating this matching in the EFT without knowing the details of the full theory. This point is studied in a simple extension of our original toy-model, by endowing the theory considered (QED in $4+\delta$ compact dimensions) with an additional fermion with mass $M_{f} \gg M_{c}$, which is eventually integrated out.

\section{CROSSING THRESHOLDS}

The simplest argument (apart from the purely dimensional ones) in favor of power-law running in theories with extra dimensions is based on the fact that in $\overline{\mathrm{MS}}$-like schemes the $\beta$ function is proportional to the number of active modes. Theories with $\delta$ extra compact dimensions contain, in general, a tower of KK modes. In particular, if we embed QED in extra dimensions we find that electrons (also photons) have a tower of KK modes with masses $M_{n}^{2}=\left(n_{1}^{2}+n_{2}^{2}+\cdots+n_{\delta}^{2}\right) M_{c}^{2}$ with $n_{i}$ integer values and $M_{c}=1 / R_{c}$ the compactification scale. The exact multiplicity of the spectrum depends on the details of the compactification procedure (torus, orbifold, etc). As soon as we cross the compactification scale, the KK modes begin to contribute, and therefore one expects that the $\beta$ function of this theory will start to receive additional contributions from them. In a general renormalization scheme 
satisfying decoupling one can naively write

$$
\beta=\sum_{n} \beta_{0} f\left(\frac{\mu}{M_{n}}\right)
$$

where $\mu$ is the renormalization scale, $\beta_{0}$ is the contribution of a single mode, and $f(\mu / M)$ is a general step-function that decouples the modes as $\mu$ crosses the different thresholds, namely $f(\mu / M) \rightarrow 0 \quad \mu \ll M$ and $f(\mu / M) \rightarrow 1 \mu \gg M$. For instance in $\overline{\mathrm{MS}}$ schemes $f(\mu / M) \equiv \theta(\mu / M-1)$ where $\theta(x)$ is the step-function. Then one finds $\left(\Omega_{\delta}=2 \pi^{\delta / 2} / \Gamma(\delta / 2)\right)$

$$
\beta=\sum_{n<\mu / M_{c}} \beta_{0} \approx \beta_{0} \int d \Omega_{\delta} n^{\delta-1} d n=\beta_{0} \frac{\Omega_{\delta}}{\delta}\left(\frac{\mu^{2}}{M_{c}^{2}}\right)^{\delta / 2}
$$

This argument, simple and compelling as it may seem, cannot be trusted completely because in $\overline{\mathrm{MS}}$ schemes the decoupling is put in by hand. Therefore, other types of schemes, in which decoupling seems natural, have been studied in the literature. For instance, in ref. [7] the VPF of the photon at $Q^{2}=0$ was calculated in the presence of the infinite tower of KK modes by using a hard cutoff in proper time, and the result was used to compute the $\beta$ function; in that case the modes decouple smoothly. In addition, after adjusting the cutoff by hand one can reproduce the aforementioned result obtained in $\overline{\mathrm{MS}}$. One can easily see that this procedure is equivalent to the use of the function $f(\Lambda / M) \equiv e^{-\frac{M_{n}^{2}}{\Lambda^{2}}}$ to decouple the KK modes

$$
\beta=\sum_{n} \beta_{0} e^{-\frac{M_{n}^{2}}{\Lambda^{2}}} \approx \beta_{0}\left(\pi \frac{\Lambda^{2}}{M_{c}^{2}}\right)^{\delta / 2} .
$$

If one chooses by hand $\mu^{\delta}=\Gamma(1+\delta / 2) \Lambda^{\delta}$, the sum in Eq. (2.3) agrees exactly with the sum obtained if one uses a sharp step-function. Even though this particular way of decoupling KK modes appears naturally in some string scenarios [4, 42, 43, 44], it hardly appears compelling from the field theory point of view; this procedure is not any better conceptually than the sharp step-function decoupling of modes: one obtains a smooth $\beta$ function because one uses a smooth function to decouple the KK modes.

These two ways of decoupling KK modes, due to the very sharp step-like behavior they impose, lead to a finite result in (2.1) for any number of extra dimensions. One is tempted to ask, however, what would happen if one were to use a more physical way of passing thresholds. In fact, heavy particles decouple naturally and smoothly in the VPF, because they cannot be produced physically. Specifically, in QED in 4-dimensions at the one-loop 
level, the imaginary part, $\Im m \Pi\left(q^{2}\right)$, of the $\operatorname{VPF} \Pi\left(q^{2}\right)$ is directly related, via the optical theorem, to the tree level cross sections $\sigma$ for the physical processes $e^{+} e^{-} \rightarrow f^{+} f^{-}$, by

$$
\Im m \Pi(s)=\frac{s}{e^{2}} \sigma\left(e^{+} e^{-} \rightarrow f^{+} f^{-}\right)
$$

Given a particular contribution to the spectral function $\Im m \Pi(s)$, the corresponding contribution to the renormalized vacuum polarization function $\Pi_{R}\left(q^{2}\right)$ can be reconstructed via a once-subtracted dispersion relation. For example, for the one-loop contribution of the fermion $f$, choosing the on-shell renormalization scheme, one finds (if $q$ is the physical momentum transfer with $q^{2}<0$, as usual we define $\left.Q^{2} \equiv-q^{2}\right)$ :

$$
\begin{gathered}
\Pi_{R}(Q)=Q^{2} \int_{4 m_{f}^{2}}^{\infty} d s \frac{1}{s\left(s+Q^{2}\right)} \frac{1}{\pi} \Im m \Pi(s) \\
=\frac{\alpha}{\pi} \times\left\{\begin{array}{lr}
\frac{1}{15} \frac{Q^{2}}{m_{f}^{2}}+\mathcal{O}\left(\frac{Q^{4}}{m_{f}^{4}}\right) & Q^{2} / m_{f}^{2} \rightarrow 0 \\
\frac{1}{3} \ln \left(\frac{Q^{2}}{m_{f}^{2}}\right)-\frac{5}{9}+\mathcal{O}\left(\frac{m_{f}^{2}}{Q^{2}}\right) & Q^{2} / m_{f}^{2} \rightarrow \infty,
\end{array}\right.
\end{gathered}
$$

where $\alpha \equiv e^{2} /(4 \pi)$. The above properties can be extended to the QCD effective charge [45], with the appropriate modifications to take into account the non-abelian nature of the theory, and provide a physical way for computing the matching equations between couplings in QCD at quark mass thresholds. One computes the VPF of QCD with $n_{f}$ flavors and that of QCD with $n_{f}-1$ flavors, and requires that the effective charge is the same for $Q^{2} \ll m_{f}$ in the two theories. This procedure gives the correct relation between the couplings in the two theories [23, 46, 47]. However, one can easily see that this cannot work for more than one extra dimension. To see that, let us consider the decoupling function $f(\mu / M)$ provided by the one-loop VPF, which, as explained, captures correctly the physical thresholds. The corresponding $f(\mu / M)$ may be obtained by differentiating $\Pi_{R}(Q)$ once with respect to $Q^{2}$; it is known [48] that the answer can be well-approximated by a simpler function of the form $f(\mu / M)=\mu^{2} /\left(\mu^{2}+5 M^{2}\right)$. We see immediately that if we insert this last function in Eq. (2.1) and perform the sum over all KK modes the result is convergent only for one extra dimension (with a coefficient which is different from the one obtained with the renormalization schemes mentioned earlier), while it becomes highly divergent for several extra dimensions. We conclude therefore that the physical way of decoupling thresholds provided by the VPF seems to lead to a divergent $\beta$ function in more than one extra dimension. As we will see, 
this is due to the fact that, in order to define properly the one-loop VPF for $\delta>1$, more than one subtraction is needed.

\section{A TOY MODEL}

To be definite we will consider a theory with one fermion and one photon in $4+\delta$ dimensions, in which the $\delta$ extra dimensions are compactified on a torus of equal radii $R_{c} \equiv 1 / M_{c}$. The Lagrangian is given by

$$
\mathcal{L}_{\delta}=-\frac{1}{4} F^{M N} F_{M N}+i \bar{\psi} \gamma^{M} D_{M} \psi+\mathcal{L}_{\mathrm{ct}}
$$

where $M=0, \cdots, 3, \cdots, 3+\delta$. We will also use greek letters to denote four-dimensional indices $\mu=0, \cdots 3 . D_{M}=\partial_{M}-i e_{D} A_{M}$ is the covariant derivative with $e_{D}$ the coupling in $4+$ $\delta$ dimensions which has dimension $\left[e_{D}\right]=1 / M^{\delta / 2}$. After compactification, the dimensionless gauge coupling in four-dimensions, $e_{4}$, and the dimensionfull $4+\delta$ coupling are related by the compactification scale ${ }^{2}$

$$
e_{4}=e_{D}\left(\frac{M_{c}}{2 \pi}\right)^{\delta / 2}
$$

Evidently $e_{D}$ is determined from the four-dimensional gauge coupling and the compactification scale, but in the uncompactified space we can regard it as a free parameter (as $f_{\pi}$ in $\chi P T)$. Finally $\mathcal{L}_{\mathrm{ct}}$ represents possible gauge invariant operators with dimension $2+D$ or higher, which are in general needed for renormalizing the theory; they can be computed only if a more complete theory, from which our effective theory originates, is given. For instance, by computing the VPF we will see that a $\mathcal{L}_{\text {ct }}$ of the form

$$
\mathcal{L}_{\mathrm{ct}}=\frac{c_{1}}{M_{s}^{2}} D_{M} F^{M K} D^{N} F_{N K}+\cdots
$$

is needed to make it finite.

The spectrum after compactification contains a photon (the zero mode of the fourdimensional components of the gauge boson), the $\delta$ extra components of the gauge boson remain in the spectrum as $\delta$ massless real scalars, a tower of massive vector bosons with masses $M_{n}^{2}=\left(n_{1}^{2}+n_{2}^{2}+\cdots+n_{\delta}^{2}\right) M_{c}^{2}, n_{i} \in \mathbb{Z}, n_{i} \neq 0,2^{[\delta / 2]}$ massless Dirac fermions (here

\footnotetext{
${ }^{2}$ Note that the factors $2 \pi$ depend on the exact way the extra dimensions are compactified (on a circle, orbifold, etc).
} 
the symbol $[x]$ represents the closest integer to $x$ smaller or equal than $x$ ), and a tower of massive Dirac fermions with masses given also by the above mass formula. Note that this theory does not lead to normal QED at low energies, first because the $\delta$ extra components of the gauge boson remain in the spectrum, and second because in $4+\delta$ dimensions the fermions have $4 \cdot 2^{[\delta / 2]}$ components, which remain as zero modes, leading at low energy to a theory with $2^{[\delta / 2]}$ Dirac fermions. In the $D=4+\delta$ theory these will arise from the trace of the identity of the $\gamma$ matrices, which just counts the number of components of the spinors. To obtain QED as a low energy one should project out the correct degrees of freedom by using some more appropriate compactification (for instance, orbifold compactifications can remove the extra components of the photon from the low energy spectrum, and leave just one Dirac fermion). However this is not important for our discussion of the VPF, we just have to remember to drop the additional factors $2^{[\delta / 2]}$ to make contact with usual QED with only one fermion. Theories of this type, with all particles living in extra dimensions are called theories with "universal extra dimensions" [49] and have the characteristic that all the effects of the KK modes below the compactification scale cancel at tree level due to the conservation of the KK numbers. In particular, and contrary to what happens in theories where gauge and scalar fields live in the bulk and fermions in the brane [50, 51], no divergences associated to summations over KK towers appear at tree level. Finally, the couplings of the electron KK modes to the standard zero-mode photon are universal and dictated by gauge invariance. The couplings among the KK modes can be found elsewhere [52, 53]; they will not be important for our discussion of the VPF that we present here.

\section{THE VACUUM POLARIZATION IN THE PRESENCE OF $K K$ MODES}

In this section we will study in detail the behavior of the one-loop VPF in the theory defined above for general values of the number $\delta$ of extra dimensions The main problems we want to address are: i) the general divergence structure of the VPF, ii) demonstrate that it is possible to regulate the UV divergences using dimensional regularization, iii) the appearance

of non-logarithmic (power) corrections, and, iv) their comparison to the analogous terms obtained when resorting to a hard-cutoff regularization. 


\section{A. The imaginary part of the vacuum polarization}

One can try to compute directly the VPF of the zero-mode photon in a theory with infinite KK fermionic modes. However, one immediately sees that, in addition to the logarithmic divergences that one finds in QED, new divergences are encountered when summing over the infinite number of KK modes. One can understand the physical origin of these divergences more clearly by resorting to the unitarity relation (here $s$ denotes the center-of-mass energy available for the production process):

$$
\begin{aligned}
\Im m \Pi^{(\delta)}(s) & =\frac{s}{e_{4}^{2}} \sum_{n} \sigma\left(e^{+} e^{-} \rightarrow f_{n}^{+} f_{n}^{-}\right) \\
& =\frac{\alpha_{4}}{3} \sum_{n<n_{\mathrm{th}}}\left(1+\frac{2 M_{n}^{2}}{s}\right) \sqrt{1-4 M_{n}^{2} / s}
\end{aligned}
$$

where $n<n_{\text {th }}$ represents the sum over all the electron KK modes such that $4\left(n_{1}^{2}+n_{2}^{2}+\cdots n_{\delta}^{2}\right) M_{c}^{2}<s$, and $\alpha_{4}=e_{4}^{2} /(4 \pi)$. This sum can be evaluated approximately for $s \gg M_{c}^{2}$ by replacing it by an integral; then we obtain

$$
\Im m \Pi^{(\delta)}(s) \approx \frac{\alpha_{4}}{2^{3+\delta}} \frac{(\delta+2) \pi^{(\delta+1) / 2}}{\Gamma((\delta+5) / 2)}\left(\frac{s}{M_{c}^{2}}\right)^{\delta / 2} .
$$

It turns out that this last result captures the behavior of the same quantity when the extra dimensions are not compact; this is so because, at high energies, the effects of the compactification can be neglected. In fact, this result may be deduced on simple dimensional grounds: as commented, the gauge coupling in $4+\delta$ dimensions has dimension $1 / M^{\delta / 2}$; therefore one expects that $\Im m \Pi^{(\delta)}(s)$ will grow with $s$ as $\left(s / M^{2}\right)^{\delta / 2}$, which is what we obtained from the explicit calculation. To see how rapidly one reaches this regime we can plot the exact result of $\Im m \Pi^{(\delta)}(s)$ together with the asymptotic value. As we can see in Fig 1, the asymptotic limit is reached very fast, especially for higher dimensions. For practical purposes one can reliably use the asymptotic value soon after passing the first threshold, $Q>2 M_{c}$, incurring errors which are below $10 \%$.

Now we can try to obtain the real part by using a dispersion relation as the one used in 4-dimensional QED, i.e. Eq.(2.5) . However, one immediately sees that it will need a number of subtractions which depends on the value of $\delta$. Thus, for just one extra dimension, as in 4-dimensional QED, one subtraction is enough, for $\delta=2$ and $\delta=3$ two subtractions are needed, and so on. This just manifests the non-renormalizability of the theory, and 

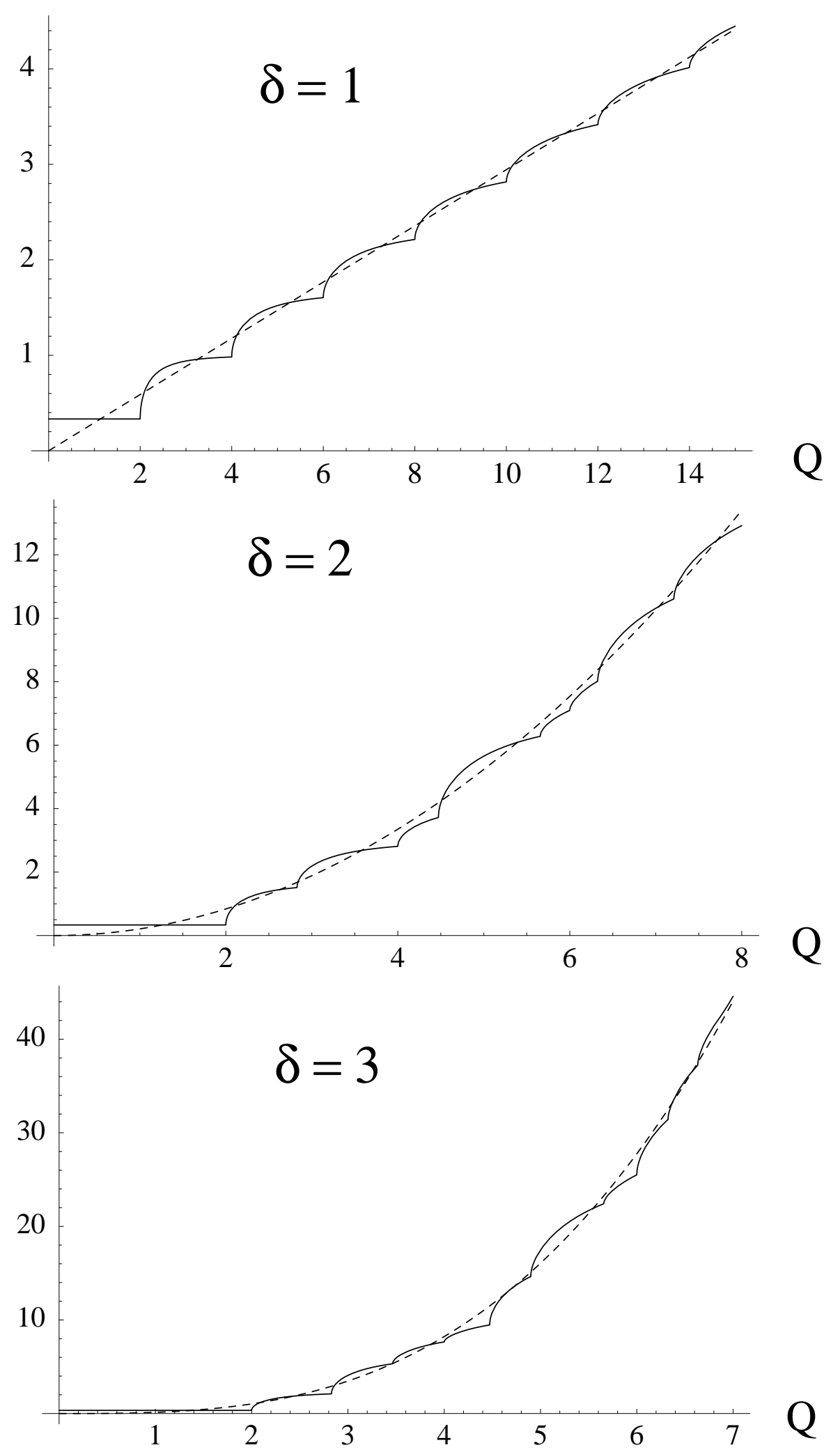

FIG. 1: $\Im m \Pi^{(\delta)}(Q)$ as compared with the asymptotic value $(\delta=1,2,3)$. $Q$ is given in units of $M_{c}$. 
in the effective field theory language, the need for higher dimension operators acting as counterterms. Even though this "absorptive" approach is perfectly acceptable, it would be preferable to have a way of computing the real part directly at the Lagrangian level (by computing loops, for instance). As commented in the introduction, to accomplish this we will use dimensional regularization.

\section{B. The vacuum polarization in uncompactified $4+\delta$ dimensions}

When using dimensional regularization to compute the VPF in uncompactified space, to be denoted $\Pi_{\mathrm{uc}}$, simple dimensional arguments suggest that one should typically obtain contributions of the form

$$
\Pi_{\mathrm{uc}}(Q) \propto e_{4}^{2}\left(2 \pi \frac{Q}{M_{c}}\right)^{\delta},
$$

since the two vertices in the loop provide a factor $e_{D}^{2}$, whose dimensions must be compensated by the only available scale in the problem, namely $Q^{2}$. In the above formula we have used the relation of Eq.(3.2) in order to trade off $e_{D}$ for $e_{4}$. The omitted coefficient in front will be generally divergent, and will be regularized by letting $\delta \rightarrow \delta-\epsilon$.

Let us compute the $\operatorname{VPF} \Pi_{\mathrm{uc}}^{M N}(q)$ in uncompactified space, assuming that, if necessary, the dimensions will be continued to complex values. We have that

$$
\Pi_{\mathrm{uc}}^{M N}(q)=i e_{D}^{2} \int \frac{d^{4+\delta} k}{(2 \pi)^{4+\delta}} \operatorname{Tr}\left\{\gamma^{M} \frac{1}{\not k} \gamma^{N} \frac{1}{\not k+\not h}\right\},
$$

which, by gauge-invariance assumes the standard form

$$
\Pi_{\mathrm{uc}}^{M N}(q)=\left(q^{2} g^{M N}-q^{M} q^{N}\right) \Pi_{\mathrm{uc}}(q)
$$

If we now were to use that, in $D$-dimensions, $\operatorname{Tr}\left[\gamma^{M} \gamma^{N}\right]=2^{[D / 2]} g^{M N}$, we would find that the low energy limit has an extra $2^{[\delta / 2]}$ factor, which, as commented, is an artifact of the torus compactification: there are $2^{[\delta / 2]}$ too many fermions in the theory. Therefore we simply drop this factor by hand. Moreover, we use Eq.(3.2) and employ the proper-time parametrization in intermediate steps, thus arriving at:

$$
\begin{aligned}
\Pi_{\mathrm{uc}}(Q) & =\frac{e_{4}^{2}}{2 \pi^{2}}\left(\frac{\pi}{M_{c}^{2}}\right)^{\delta / 2} \int_{0}^{1} d x x(1-x) \int_{0}^{\infty} \frac{d \tau}{\tau^{1+\frac{\delta}{2}}} \exp \left\{-\tau x(1-x) Q^{2}\right\} \\
& =\frac{e_{4}^{2}}{2 \pi^{2}} \frac{\pi^{\delta / 2} \Gamma^{2}\left(2+\frac{\delta}{2}\right)}{\Gamma(4+\delta)} \Gamma\left(-\frac{\delta}{2}\right)\left(\frac{Q^{2}}{M_{c}^{2}}\right)^{\delta / 2} .
\end{aligned}
$$


A simple check of this result may be obtained by computing its imaginary part. To that end we let $Q^{2} \rightarrow-q^{2}-i \epsilon$ with $q^{2}>0$. Then

$$
\Im m\left\{-q^{2}-i \epsilon\right\}^{\delta / 2}=-\left(q^{2}\right)^{\delta / 2} \sin \frac{\delta \pi}{2} .
$$

Now we can use that $\Gamma(-\delta / 2) \Gamma(1+\delta / 2)=-\pi / \sin (\delta \pi / 2)$ to write

$$
\Im m\left\{\Pi_{\mathrm{uc}}(q)\right\}=\alpha_{4} \frac{2 \pi^{\delta / 2} \Gamma^{2}\left(2+\frac{\delta}{2}\right)}{\Gamma(4+\delta) \Gamma(1+\delta / 2)}\left(\frac{q^{2}}{M_{c}^{2}}\right)^{\delta / 2}=\frac{\alpha_{4}}{2^{3+\delta}} \frac{(\delta+2) \pi^{(\delta+1) / 2}}{\Gamma((\delta+5) / 2)}\left(\frac{q^{2}}{M_{c}^{2}}\right)^{\delta / 2}
$$

which agrees with our previous result of Eq.(4.2).

For odd values of $\delta$, the one-loop $\Pi_{\mathrm{uc}}(Q)$ computed above is finite, since the $\Gamma\left(-\frac{\delta}{2}\right)$ can be calculated by analytic continuation. This result is in a way expected, since in odd number of dimensions, by Lorentz invariance, there are no appropriate gauge invariant operators able to absorb any possible infinities generated in the one-loop VPF; this would require operators which give contributions that go like $Q^{\delta}$. Notice, however, that at higher orders $\Pi_{\mathrm{uc}}(Q)$ will eventually become divergent. For instance, in five dimensions at two loops, the VPF should go as $Q^{2}$, since there are four elementary vertices. The divergences generated by these contributions could be absorbed in an operator such as the one considered in the previous section, namely $D_{M} F^{M K} D^{N} F_{N K}$. On the other hand, when $\delta$ is even, $\Gamma\left(-\frac{\delta}{2}\right)$ has a pole, and subtractions are needed already at one loop. To compute the divergent and finite parts in a well-defined way we will use dimensional regularization, i.e. we will assume that $\delta \rightarrow \delta-\epsilon$. Notice however that, unlike in 4-dimensions, we not need to introduce an additional scale at this point, i.e. the equivalent of the 't Hooft mass scale $\mu$ : $M_{c}$ plays the role of $\mu$, and can be used to keep $e_{4}$ dimensionless. After expanding in $\epsilon$ we find a simple pole accompanied by the usual logarithm

$$
\Pi_{\mathrm{uc}}(Q) \propto\left(\frac{Q^{2}}{M_{c}^{2}}\right)^{\delta / 2}\left\{-\frac{2}{\epsilon}+\ln \left(Q^{2} / M_{c}^{2}\right)+\cdots\right\} .
$$

Here the ellipses represent a finite constant. Now, to renormalize this result we must introduce higher dimension operators (for instance, if $\delta=2$ the operator $D_{M} F^{M K} D^{N} F_{N K}$ will do the job) which could absorb the divergent piece. The downside of this, however, is that we also have to introduce an arbitrary counterterm, $\kappa$, corresponding to the contribution of the higher dimension operator; thus we obtain a finite quantity proportional to $\log \left(Q^{2} / M_{c}^{2}\right)+\kappa$. Note that, since $\kappa$ is arbitrary, we can always introduce back a renormalization scale and 
write $\log \left(Q^{2} / M_{c}^{2}\right)+\kappa=\log \left(Q^{2} / \mu^{2}\right)+\kappa(\mu)$ with $\kappa(\mu)=\kappa+\log \left(\mu^{2} / M_{c}^{2}\right)$. It is also important to remark that, in the case of odd number of dimensions, although at one loop we do not need any counterterm to make the VPF finite, higher dimensional operators could still be present and affect its value.

In the case of uncompactified space, it is interesting to compare the above result with that obtained by regularizing the integral using a hard cutoff. To study this it is enough to carry out the integral of Eq. (4.4), with a cutoff in $\tau_{0}=1 / \Lambda^{2}$ :

$$
\Pi_{\mathrm{uc}}(Q)=\frac{e_{4}^{2}}{2 \pi^{2}}\left(\frac{\pi}{M_{c}^{2}}\right)^{\delta / 2} \int_{0}^{1} d x x(1-x) \int_{\tau_{0}}^{\infty} \frac{d \tau}{\tau^{1+\delta / 2}} \exp \left\{-\tau x(1-x) Q^{2}\right\}
$$

Then, for $\delta=1,2,3$ we obtain

$$
\begin{gathered}
\Pi_{\mathrm{uc}}^{(1)}(Q)=\frac{e_{4}^{2}}{2 \pi^{2}}\left(-\frac{3 \pi^{2} Q}{64 M_{c}}+\frac{\sqrt{\pi} Q^{2}}{15 M_{c} \Lambda}+\frac{\sqrt{\pi} \Lambda}{3 M_{c}}\right), \\
\Pi_{\mathrm{uc}}^{(2)}(Q)=\frac{e_{4}^{2}}{2 \pi^{2}}\left(\frac{\pi \Lambda^{2}}{6 M_{c}^{2}}+\frac{\pi Q^{2}}{30 M_{c}^{2}}\left(\log \left(Q^{2} / \Lambda^{2}\right)+\gamma-\frac{77}{30}\right)\right), \\
\Pi_{\mathrm{uc}}^{(3)}(Q)=\frac{e_{4}^{2}}{2 \pi^{2}}\left(\frac{5 \pi^{3} Q^{3}}{768 M_{c}^{3}}-\frac{\pi^{3 / 2} Q^{2} \Lambda}{15 M_{c}^{3}}+\frac{\pi^{3 / 2} \Lambda^{3}}{9 M_{c}^{3}}\right) .
\end{gathered}
$$

As we can see, the pieces which are independent of the cutoff are exactly the same ones we obtained using dimensional regularization. But, in addition, we obtain a series of contributions which depend explicitly on the cutoff. For instance we find corrections to the gauge coupling which behave as $\Lambda^{\delta}$, and just redefine the gauge coupling we started with [54]. In the case of 5 dimensions we also generate a term linear in $Q^{2}$; however it is suppressed by $1 / \Lambda$, and therefore it approaches zero for large $\Lambda$. In the case of 6 dimensions we obtain the same logarithmic behavior we found with dimensional regularization, and the result can be cast in identical form, if the cutoff is absorbed in the appropriate counterterm. For 7 dimensions we also find divergent contributions which go as $Q^{2}$. This means that, when using cutoffs, higher dimension operators in the derivative expansion (e.g. operators giving contributions as $Q^{2}$ or higher) are necessary to renormalize the theory and must be included. In the case of dimensional regularization this type of operators is not strictly needed at one loop; however, nothing forbids them in the Lagrangian, and they could appear as "finite counterterms". If one were to identify the $\Lambda$ in the above expressions with a physical cutoff, one might get the impression that, contrary to the dimensional regularization approach where 
arbitrary counterterms are needed, one could now obtain all types of contributions with only one additional parameter, namely $\Lambda$. This is however not true: the regulator function is arbitrary, we simply have chosen one among an infinity of possibilities. By changing the regulator function we can change the coefficients of the different contributions at will, except for those few contributions which are independent of $\Lambda$. These latter are precisely the ones we have obtained by using dimensional regularization. Thus, even when using cutoffs one has to add counterterms from higher dimension operators, absorb the cutoff, and express the result in terms of a series of unknown coefficients. The lesson is that with dimensional regularization we obtain all calculable pieces, while the non-calculable pieces are related to higher dimensional terms in the Lagrangian.

What we will demonstrate next is that the one-loop VPF in the compactified theory on a torus can be renormalized exactly as the VPF in the uncompactified theory; this will allow us to compute it for any number of dimensions, and examine its behavior for large and for small values of the $Q^{2}$.

\section{The vacuum polarization in $\delta$ compact dimensions}

From the four-dimensional point of view the vacuum polarization tensor in the compactified theory is

$$
\Pi^{\mu \nu}\left(q^{2}\right)=\sum_{n} i e_{4}^{2} \int \frac{d^{4} k}{(2 \pi)^{4}} \operatorname{Tr}\left\{\gamma^{\mu} \frac{1}{\not k-m_{n}} \gamma^{\nu} \frac{1}{\not k+\not t-m_{n}}\right\}
$$

with $m_{n}^{2}=\left(n_{1}^{2}+n_{2}^{2}+\cdots+n_{\delta}^{2}\right) M_{c}^{2}$; for simplicity we have assumed a common compactification radius $R=1 / M_{c}$ for all the extra dimensions. The sum over $n$ denotes collectively the sum over all the modes $n_{i}=-\infty, \cdots,+\infty$. The contribution of each mode to this quantity seems quadratically divergent, like in ordinary QED; however, we know that gauge invariance converts it to only logarithmically divergent. But, in addition, the sum over all the modes makes the above expressions highly divergent. Instead of attempting to compute it directly, we will add and subtract the contribution of the vacuum polarization function of the uncompactified theory in $4+\delta$ dimensions:

$$
\Pi^{\mu \nu}(q)=\left[\Pi^{\mu \nu}(q)-\Pi_{\mathrm{uc}}^{\mu \nu}(q)\right]+\Pi_{\mathrm{uc}}^{\mu \nu}(q)=\Pi_{\mathrm{fin}}^{\mu \nu}(q)+\Pi_{\mathrm{uc}}^{\mu \nu}(q) .
$$

Here we have taken already into account the relation between the coupling in $4+\delta$ dimensions

and the four-dimensional coupling and have restricted the external Lorentz indices to the 
4-dimensional ones. Depending on the value of $\delta$ the vacuum polarization can be highly divergent (naively as $\Lambda^{\delta+2}$, and after taking into account gauge invariance as $\Lambda^{\delta}$ ). However, we can use dimensional regularization (or any other regularization scheme) to make it finite. The important point is that the quantity $\Pi_{\text {fin }}^{\mu \nu}(Q)$ is UV and IR finite and can unambiguously computed.

Instead of doing the two calculations from scratch, we will do the following:

i) We will first compute the compactified expression by using Schwinger's proper time, $\tau$, to regularize the UV divergences.

ii) We will show that the UV behavior of the compactified theory, $\tau \rightarrow 0$, is just the behavior of the uncompactified theory.

iii) Therefore, to compute $\Pi_{\text {fin }}^{\mu \nu}(Q)$ it is sufficient to compute $\Pi^{\mu \nu}(Q)$ and then subtract its most divergent contribution when $\tau \rightarrow 0$. We will see that it is sufficient to make it finite.

After a few manipulations $\Pi^{\mu \nu}(Q)$ can be written as

$$
\Pi^{\mu \nu}(q)=\left(q^{2} g^{\mu \nu}-q^{\mu} q^{\nu}\right) \Pi(q)
$$

where [7]

$$
\Pi(Q)=\frac{e_{4}^{2}}{2 \pi^{2}} \sum_{n} \int_{0}^{1} d x x(1-x) \int_{0}^{\infty} \frac{d \tau}{\tau} \exp \left\{-\tau\left(x(1-x) Q^{2}+m_{n}^{2}\right)\right\} .
$$

$\Pi(Q)$ can be written in terms of the function

$$
\bar{\theta}_{3}(\tau) \equiv \sum_{n=-\infty}^{+\infty} e^{-n^{2} \tau}=\sqrt{\frac{\pi}{\tau}} \bar{\theta}_{3}\left(\frac{\pi^{2}}{\tau}\right)
$$

as

$$
\Pi(Q)=\frac{e_{4}^{2}}{2 \pi^{2}} \int_{0}^{1} d x x(1-x) \int_{0}^{\infty} \frac{d \tau}{\tau} \exp \left\{-\tau x(1-x) \frac{Q^{2}}{M_{c}^{2}}\right\} \bar{\theta}_{3}^{\delta}(\tau),
$$

where we have rescaled $\tau$ in order to remove $M_{c}$ from the $\bar{\theta}_{3}(\tau)$ function. This last expression for $\Pi(Q)$ is highly divergent in the $\operatorname{UV}(\tau \rightarrow 0)$, because in that limit the $\bar{\theta}_{3}(\tau)$ function goes as $\sqrt{\pi / \tau}$. Then, if we define, as in Eq. (4.10), $\Pi_{\mathrm{f} i n}=\Pi-\Pi_{\mathrm{uc}}$, we have

$$
\Pi_{\text {fin }}(Q)=\frac{e_{4}^{2}}{2 \pi^{2}} \int_{0}^{1} d x x(1-x) \int_{0}^{\infty} \frac{d \tau}{\tau} \exp \left\{-\tau x(1-x) \frac{Q^{2}}{M_{c}^{2}}\right\}\left(\bar{\theta}_{3}^{\delta}(\tau)-\left(\frac{\pi}{\tau}\right)^{\delta / 2}\right)
$$

which is completely finite for any number of dimensions. In fact, the last term provides a factor

$$
F_{\delta}(\tau) \equiv \bar{\theta}_{3}^{\delta}(\tau)-\left(\frac{\pi}{\tau}\right)^{\delta / 2} \stackrel{\tau \rightarrow 0}{\longrightarrow} 2 \delta\left(\frac{\pi}{\tau}\right)^{\delta / 2} \exp \left\{-\frac{\pi^{2}}{\tau}\right\}
$$




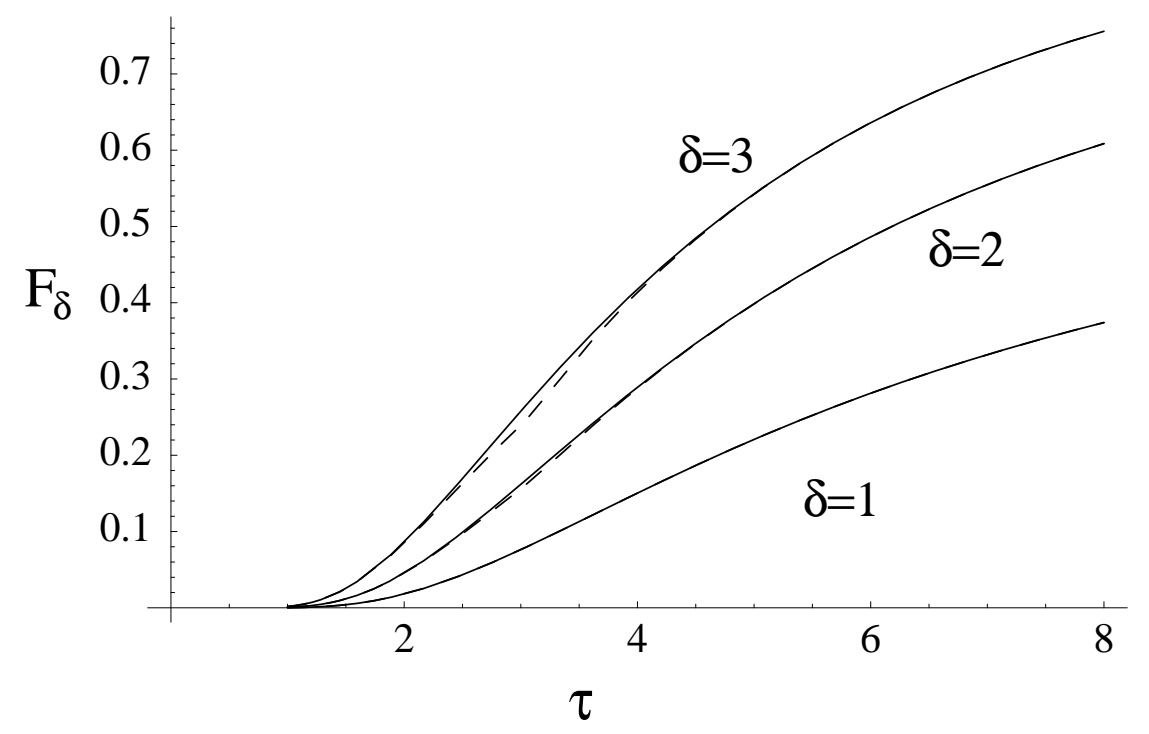

FIG. 2: Exact values of $F_{\delta}(\tau)$ (solid) as compared with the approximation discussed in the text (dashed) for $\delta=1,2,3$.

that makes the integral convergent in the UV, while for large $\tau$ this function goes to 1 quite fast. In this region the integral is cut off by the exponential of momenta; so we can think of the exponential $\exp \left\{-\tau x(1-x) \frac{Q^{2}}{M_{c}^{2}}\right\}$ as providing a cutoff for $\tau>4 M_{c}^{2} / Q^{2}$, and $F_{\delta}(\tau)$ as providing a cutoff for $\tau<\pi^{2}$. With this in mind, we can estimate $\Pi_{\text {fin }}(Q)$ as

$$
\Pi_{\mathrm{fin}}(Q) \approx \frac{e_{4}^{2}}{2 \pi^{2}} \sum_{n} \int_{0}^{1} d x x(1-x) \int_{\pi^{2}}^{4 M_{c}^{2} / Q^{2}} \frac{d \tau}{\tau}=-\frac{e^{2}}{2 \pi^{2}} \frac{1}{6} \log \frac{Q^{2} \pi^{2}}{4 M_{c}^{2}}, \quad Q^{2}<4 M_{c}^{2} / \pi^{2}
$$

it is just the ordinary running of the zero mode. As $Q^{2}$ grows, the upper limit of integration is smaller than the lower limit, and then we expect that $\Pi_{\text {fin }}(Q)$ should vanish. In that region $\Pi(Q)$ will be dominated completely by $\Pi_{\mathrm{uc}}(Q)$.

Let us evaluate $\Pi_{\text {fin }}(Q)$ for any number of extra dimensions. To this end we will approximate the function $F_{\delta}(\tau)$ as follows

$$
F_{\delta}(\tau)=\left\{\begin{array}{l}
2 \delta\left(\frac{\pi}{\tau}\right)^{\delta / 2} \exp \left\{-\frac{\pi^{2}}{\tau}\right\} \quad \tau<\pi \\
1+2 \delta \exp \{-\tau\}-\left(\frac{\pi}{\tau}\right)^{\delta / 2} \quad \tau>\pi
\end{array} .\right.
$$

The matching point in $\tau=\pi$ makes the function continuous. In Fig. 2 we display the exact function $F_{\delta}(\tau)$ (solid) and the approximation above (dashed) for $\delta=1,2,3$. The approximation is very good except at a small region around the matching point $\tau=\pi$. This can be further improved by adding more terms from the expansions of the $\bar{\theta}(\tau)$ functions. 
The approximate expression of Eq.(4.12) can be used to obtain semi-analytical expansions for $\Pi_{\text {fin }}^{\delta}(Q)$ for small $Q^{2}$ (we define $w \equiv Q^{2} / M_{c}^{2}$ )

$$
\begin{aligned}
& \Pi_{\text {fin }}^{(1)}(Q)=\frac{e_{4}^{2}}{2 \pi^{2}}(-0.335-0.167 \log (w)+0.463 \sqrt{w}-0.110 w+\cdots) \\
& \Pi_{\text {fin }}^{(2)}(Q)=\frac{e_{4}^{2}}{2 \pi^{2}}(-0.159-0.167 \log (w)-0.105 w(\log (w)-1.75)+\cdots), \\
& \Pi_{\text {fin }}^{(3)}(Q)=\frac{e_{4}^{2}}{2 \pi^{2}}\left(-0.0937-0.167 \log (w)+0.298 w-0.202 \sqrt{w^{3}}+\cdots\right) .
\end{aligned}
$$

To see how good these approximate results are, we can compare with the exact results that can be obtained when $\delta=1$. In this case we have

$$
\begin{gathered}
\Pi_{\mathrm{fin}}^{(1)}(Q)=\frac{e_{4}^{2}}{2 \pi^{2}} \int_{0}^{1} d x x(1-x) \sum_{n=1}^{\infty} \int_{0}^{\infty} \frac{d \tau}{\tau} \exp \{-\tau x(1-x) w\} 2\left(\frac{\pi}{\tau}\right)^{1 / 2} \exp \left\{-\frac{n^{2} \pi^{2}}{\tau}\right\} \\
=\frac{e_{4}^{2}}{2 \pi^{2}} \int_{0}^{1} d x x(1-x) \sum_{n=1}^{\infty} \frac{2}{n} \exp \{-2 \pi n \sqrt{x(1-x) w}\} \\
=\frac{e_{4}^{2}}{2 \pi^{2}} \int_{0}^{1} d x x(1-x)(-2 \log (1-\exp (-2 \pi \sqrt{x(1-x) w})))
\end{gathered}
$$

This last expression can be expanded for $w \ll 1$, and the integral over $x$ can then be performed analytically, yielding

$$
\Pi_{\mathrm{fin}}^{(1)}(Q) \approx \frac{e_{4}^{2}}{2 \pi^{2}}\left(\frac{1}{18}(5-6 \log (2 \pi))-\frac{1}{6} \log w+\frac{3 \pi^{2}}{64} \sqrt{w}-\frac{\pi^{2}}{90} w+\cdots\right)
$$

which is in excellent agreement with our approximation (see Eq. (4.13)).

Eq. (4.16) can also be used to obtain the behavior of $\Pi_{\text {fin }}^{(1)}(Q)$ for $w \gg 1$. In this limit we obtain

$$
\Pi_{\text {fin }}^{(1)}(Q) \approx \frac{e_{4}^{2}}{2 \pi^{2}} \frac{3 \zeta(5)}{\pi^{4}} \frac{M_{c}^{4}}{Q^{4}}, \quad Q^{2} \gg M_{c}^{2}
$$

For higher dimensions things are more complicated, but the behavior is the same, and we find

$$
\Pi_{\text {fin }}^{(\delta)}(Q) \approx \frac{e_{4}^{2}}{2 \pi^{2}} \frac{4 \delta \Gamma(2+\delta / 2)}{\pi^{4+\delta / 2}} K_{\delta} \frac{M_{c}^{4}}{Q^{4}}, \quad Q^{2} \gg M_{c}^{2},
$$

where $K_{\delta}$ is of the order of unity and is determined numerically $\left(K_{1}=\zeta(5)=1.037, K_{2}=\right.$ $\left.1.165, K_{3}=1.244\right)$. However, since the uncompactified contribution grows as $\left(Q^{2} / M_{c}^{2}\right)^{\delta / 2}$ it is obvious that the contributions to $\Pi^{(\delta)}(Q)$ from $\Pi_{\text {fin }}^{(\delta)}(Q)$ will be completely irrelevant for $Q^{2} \gg M_{c}^{2}$ 
Adding the finite and the uncompactified contributions we find that for $Q^{2} \ll M_{c}^{2}$ the uncompactified contribution exactly cancels the corresponding piece obtained from the expansion of $\Pi_{\text {fin }}^{(\delta)}(Q)$ (the $\sqrt{w}$ piece for $\delta=1$, the $w \log (w)$ piece for $\delta=2$, or the $\sqrt{w^{3}}$ for $\delta=3)$. Then, for $Q^{2} \ll M_{c}^{2}$ and choosing $\mu=M_{c}$ we finally find:

$$
\Pi^{(\delta)}(Q)=\frac{e_{4}^{2}}{2 \pi^{2}}\left(a_{0}^{(\delta)}-\frac{1}{6} \log \left(\frac{Q^{2}}{M_{c}^{2}}\right)+a_{1}^{(\delta)} \frac{Q^{2}}{M_{c}^{2}}+\cdots\right), \quad Q^{2} \ll M_{c}^{2}
$$

with the coefficients for 1,2 and 3 extra dimensions given by

\begin{tabular}{|c|c|c|c|}
\hline$\delta$ & 1 & 2 & 3 \\
\hline$a_{0}^{(\delta)}$ & -0.335 & -0.159 & -0.0937 \\
\hline$a_{1}^{(\delta)}$ & -0.110 & 0.183 & 0.298 \\
\hline
\end{tabular}

As we will see below, in general the coefficients $a_{1}^{(\delta)}$ can be affected by non-calculable contributions from higher dimension operators in the effective Lagrangian, which we have not included.

The following comments related to Eq. (4.18), which is only valid in the dimensional regularization scheme we are using, are now in order:

(i) From Eq. (4.18) we see that for small $Q^{2}$, as expected, we recover the standard logarithm with the correct coefficient, independently of the number of extra dimensions. In addition, interestingly enough, we can compute also the constant term. Thus, although the full theory in $4+\delta$ dimensions is non-renormalizable and highly divergent, the low energy limit of the VPF calculated in our dimensional regularization scheme is actually finite: when seen from low energies the compactified extra dimensions seem to act as an ultraviolet regulator for the theory.

(ii) When the energy begins to grow, we start seeing effects suppressed by $Q^{2} / M_{c}^{2}$, which are finite, at one loop, for any number of dimensions except for $\delta=2$. This is so because the gauge couplings have dimensions $1 / M^{\delta / 2}$, and therefore, the one-loop VPF goes like $1 / M^{\delta}$.

(iii) For $\delta=1$ one finds that, because of gauge and Lorentz invariance, there are no possible counterterms of this dimension. The VPF must be finite, and that is precisely the result one obtains with dimensional regularization. This of course changes if higher loops are considered: for instance, two-loop diagrams go like $1 / M^{2}$, and, in general, we expect that they will have divergences, which, in turn, should be absorbed in the appropriate counterterms. In principle the presence of these counterterms could pollute our result; 
however, the natural size of these counterterms, arising at two loops, should be suppressed compared to the finite contributions we have computed.

(iv) For $\delta=2$ one finds that the VPF goes as $1 / M^{2}$, already at one loop, and that the result is divergent. The divergences have to be absorbed in the appropriate counterterm coming from higher dimension operators in the higher dimensional theory. The immediate effect of this, is that the coefficient of the $Q^{2}$ term in $\Pi^{(2)}(Q)$ becomes arbitrary, its value depending on the underlying physics beyond the compactification scale.

(v) For $\delta>2$ all loop contributions to the $Q^{2}$ term are finite, simply because of the dimensionality of the couplings. This, however, does not preclude the existence of finite counterterms, which could be generated by physics beyond the compactification scale, that is, contributions from operators suppressed by two powers of the new physics scale like the operator in Eq. (3.3.3).

For $Q^{2} \gg M_{c}^{2}$ the full VPF is completely dominated by the uncompactified contribution:

$$
\begin{aligned}
& \Pi^{(1)}(Q)=-\frac{e_{4}^{2}}{2 \pi^{2}} \frac{3 \pi^{2}}{64} \sqrt{\frac{Q^{2}}{M_{c}^{2}}}, \\
& \Pi^{(2)}(Q)=\frac{e_{4}^{2}}{2 \pi^{2}} \frac{\pi}{30} \frac{Q^{2}}{M_{c}^{2}} \log \left(\frac{Q^{2}}{M_{c}^{2}}\right) \quad Q^{2} \gg M_{c}^{2}, \\
& \Pi^{(3)}(Q)=\frac{e_{4}^{2}}{2 \pi^{2}} \frac{5 \pi^{3}}{768}\left(\frac{Q^{2}}{M_{c}^{2}}\right)^{\frac{3}{2}} .
\end{aligned}
$$

As before, the VPF could also receive non-calculable contributions from higher dimension operators which we have not included; in fact, for $\delta=2$, these are needed to renormalize the VPF. How large can these non-calculable contributions be? Since our D-dimensional theory is an effective theory valid only for $Q^{2} \ll M_{s}^{2}$, even above $M_{c}$ the results will be dominated by the lowest power of $Q^{2}$. In the case of $\delta=1$, the first operator that one can write down goes as $Q^{2}$; therefore we expect that the one-loop contribution, of order $\sqrt{Q^{2}}$, that we have computed, will dominate completely the result, as long as we do not stretch it beyond the applicability of the effective Lagrangian approach. For $\delta=2$, counterterms are certainly needed at order $Q^{2}$; still one can hope that the result will be dominated by the logarithm (as happens with chiral logarithms in $\chi P T$ ). For $\delta=3$ (and higher), the one loop result grows as $\left(Q^{2}\right)^{\delta / 2}$; however there could be operators giving contributions of order $Q^{2}$ with unknown coefficients (in fact although in dimensional regularization those are not needed, they must 
be included if cutoffs are used to regularize the theory). Therefore, unless for some reason they are absent from the theory, the result will be dominated by those operators.

\section{MATCHING OF GAUGE COUPLINGS}

Using the VPF constructed in the previous section we can define a higher dimensional analogue of the conventional QED effective charge [55, 56], which will enter in any process involving off-shell photons, e.g.

$$
\left.\frac{1}{\alpha_{\mathrm{eff}}(Q)} \equiv \frac{1}{\alpha_{4}}\left(1+\Pi^{(\delta)}(Q)\right)\right|_{\overline{\mathrm{MS}}_{\delta}},
$$

where $\alpha_{4}=e_{4}^{2} /(4 \pi)$. We remind the reader that $e_{4}$ denotes the (dimensionless) coupling of the four-dimensional theory including all KK modes; it is directly related to the gauge coupling in the theory with $\delta$ extra dimensions by Eq.(3.2). The subscript $\overline{\mathrm{MS}}_{\delta}$ means that the VPF has been regularized using dimensional regularization in $D=4+\delta-\epsilon$ dimensions, and that divergences, when present, are subtracted according to the $\overline{\mathrm{MS}}$ procedure.

To determine the relation between $\alpha_{4}$ and the low energy coupling in QED, we have to identify the effective charge computed in the compactified theory with the low energy effective charge, at some low energy scale (for instance $Q^{2}=m_{Z}^{2} \ll M_{c}^{2}$ ), where both theories are valid. In that limit we can trust our approximate results of Eq. (4.18), and write

$$
\frac{1}{\alpha_{\mathrm{eff}}\left(m_{Z}\right)}=\frac{1}{\alpha_{4}}+\frac{2}{\pi} a_{0}^{(\delta)}-\frac{2}{3 \pi} \log \left(\frac{m_{Z}}{M_{c}}\right) .
$$

This equation connects the low energy QED coupling with the coupling in the compactified D-dimensional theory, regularized by dimensional regularization. Note that this equation is completely independent of the way subtractions are performed to remove the poles in $1 / \epsilon$. These poles only appear (and only for even number of dimensions) in the contributions proportional to $Q^{\delta}$, which vanish for $Q \rightarrow 0$. Eq. (5.2) contains, apart from a finite constant, the standard logarithmic running from $m_{Z}$ to the compactification scale $M_{c}$. It is interesting to notice that, in this approach, the logarithm comes from the finite piece, and should therefore be considered as an infrared (IR) logarithm. When seen from scales smaller than $M_{c}$, these logarithms appear to have an UV origin, while, when seen from scales above $M_{c}$, appear as having an IR nature. 
It is important to emphasize that, in this scheme, the gauge coupling does not run any more above the compactification scale. This seems counter-intuitive, but it is precisely what happens in $\chi P T$ when using dimensional regularization: $f_{\pi}$ does not run, it just renormalizes higher dimensional operators [26].

Now we can use Eq. (5.2) to write the effective charge at all energies in terms of the coupling measured at low energies:

$$
\frac{1}{\alpha_{\mathrm{eff}}(Q)} \equiv \frac{1}{\alpha_{\mathrm{eff}}\left(m_{Z}\right)}+\left.\frac{1}{\alpha_{4}}\left(\Pi^{(\delta)}(Q)-\Pi^{(\delta)}\left(m_{Z}\right)\right)\right|_{\overline{\mathrm{MS}}_{\delta}} .
$$

Note that the last term is independent of $\alpha_{4}$ due to the implicit dependence of $\Pi^{(\delta)}$ on it. Eq. (5.3) has the form of a momentum-subtracted definition of the coupling; in fact, in four dimensions it is just the definition of the momentum-subtracted running coupling. For $\delta=1$ and at one loop, $\Pi^{(\delta)}(Q)-\Pi^{(\delta)}\left(m_{Z}\right)$ is finite, and $\alpha_{\text {eff }}(Q)$ can still be interpreted as a momentum-subtracted definition of the coupling. For $\delta>1$, however, Eq. (5.3) involves additional subtractions, a fact which thwarts such an interpretation.

For $Q^{2} \ll M_{c}^{2}$ we can expand $\Pi^{(\delta)}(Q)$ and obtain

$$
\frac{1}{\alpha_{\mathrm{eff}}(Q)} \equiv \frac{1}{\alpha_{\mathrm{eff}}\left(m_{Z}\right)}-\frac{2}{3 \pi} \log \left(\frac{Q}{m_{Z}}\right)+\mathcal{O}\left(\frac{Q^{2}}{M_{c}^{2}}\right),
$$

which is nothing but the standard expression of the effective charge in QED, slightly modified by small corrections of order $Q^{2} / M_{c}^{2}$. However, as soon as $Q^{2} / M_{c}^{2}$ approaches unity, the effects of the compactification scale start to appear in $\alpha_{\text {eff }}(Q)$, forcing it to deviate dramatically from the logarithmic behavior, as shown in Fig. 3 ,

The crucial point, however, is that this effective charge cannot be interpreted anymore as the running coupling (as can be done in four dimensions) since it may receive contributions from higher dimension operators; in fact some of them are needed to define this quantity properly. These contributions have nothing to do with the gauge coupling which is defined as the coefficient of the operator $F^{2}$. In particular, one should not use this quantity to study gauge coupling unification. Instead, one could use Eq. (5.21), which relates the coupling measured at low energies with the one appearing in the $D$-dimensional Lagrangian valid at energies $M_{c}<Q<M_{s}$. This relation involves a logarithmic correction, which is the only contribution that can be reliably computed without knowing the physics beyond $M_{s}$.

It is instructive to see what happens if instead of dimensional regularization we use hard cutoffs to regularize the uncompactified part of the VPF as in Eqs. (4.6)-(4.9). Then, when 


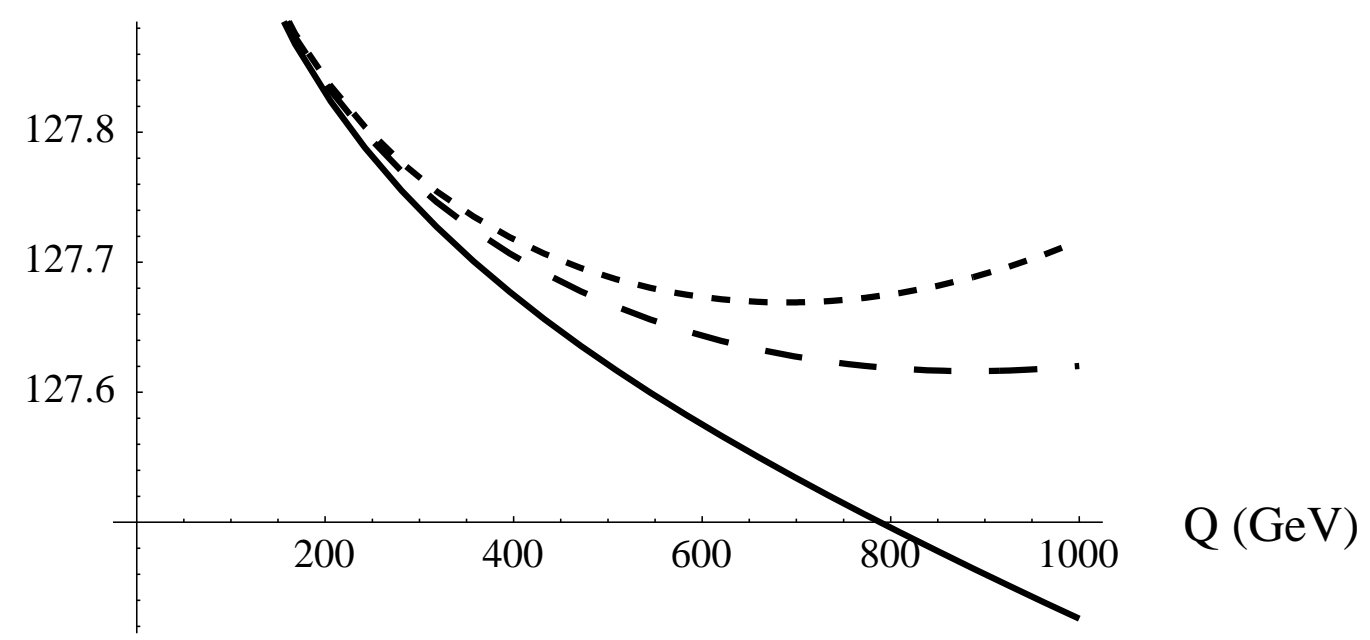

FIG. 3: The "effective charge" against the energy scale for $\delta=1$ (solid), $\delta=2$ (short dash), $\delta=3$ (long dash).

using cutoffs, one can define an "effective charge" as in Eq. (5.1)

$$
\left.\frac{1}{\alpha_{\mathrm{eff}}(Q)} \equiv \frac{1}{\alpha_{4}(\Lambda)}\left(1+\Pi^{(\delta)}(Q)\right)\right|_{\Lambda},
$$

where $\alpha_{4}(\Lambda)$ now is the coupling constant in the theory regularized with cutoffs and the subscript $\Lambda$ indicates that the VPF has been regularized with cutoffs. The use of a $\Lambda$ dependent coupling obviously implies the WEFT formulation, in which the cutoff is not removed from the theory. On the other hand, in the CEFT formulation one should renormalize the coupling constant by adding the appropriate counterterms and then take the limit $\Lambda \rightarrow \infty$. This usually brings in a new scale at which the coupling is defined, and which effectively replaces $\Lambda$ in the previous equation. Notice also that for $\delta=2$ in Eq. (4.8) there are $\log$ arithmic contributions proportional to $Q^{2}$, which cannot be removed when $\Lambda \rightarrow \infty$. The same is true for $\delta>2$, but with dependencies which are proportional to $\Lambda^{(\delta-2)}$. This just manifests the need of higher dimensional operators, as was already clear in the dispersive approach, to define properly the effective charge. As one can see, the full VPF contains a term that goes as $\Lambda^{\delta}$ and is independent of $Q$. This piece survives when $Q \rightarrow 0$, and thus we obtain (we assume $m_{Z}^{2} \ll Q^{2}$ )

$$
\frac{1}{\alpha_{\mathrm{eff}}\left(m_{Z}\right)}=\frac{1}{\alpha_{4}(\Lambda)}+\frac{2}{3 \pi \delta}\left(\sqrt{\pi} \frac{\Lambda}{M_{c}}\right)^{\delta}+\frac{2}{\pi} a_{0}^{(\delta)}-\frac{2}{3 \pi} \log \left(\frac{m_{Z}}{M_{c}}\right) .
$$

Since $\alpha_{\text {eff }}\left(m_{Z}\right)$ should be the same in the two schemes, we find the following relation between 
$\alpha_{4}$ and $\alpha_{4}(\Lambda)$

$$
\frac{1}{\alpha_{4}}=\frac{1}{\alpha_{4}(\Lambda)}+\frac{2}{3 \pi \delta}\left(\sqrt{\pi} \frac{\Lambda}{M_{c}}\right)^{\delta}
$$

If one identifies $\Lambda$ with the onset of a more complete theory beyond the compactification scale, but at which the EFT treatment is still valid, i.e. if one assumes that $\Lambda \sim M_{G} \ll M_{s}$, $M_{G}$ being this new scale, Eq.(5.6) could be reinterpreted as a matching equation between the coupling $\alpha_{4}$ of our effective theory and the coupling of the theory at scales $M_{G}, \alpha_{4}\left(M_{G}\right)$. Eq. (5.6) generically tells us that one expects corrections which go as $\left(M_{G} / M_{c}\right)^{\delta}$. However, without knowledge of the full theory beyond $M_{G}$, the meaning of $M_{G}$ (or even $\alpha_{4}\left(M_{G}\right)$ ) is unclear. In particular, if the new theory is some Grand Unified Theory in extra dimensions, $M_{G}$ will be, in general, not just one single mass, but several masses of the same order of magnitude, related by different coefficients. In the case of logarithmic running those coefficients can be neglected, because they give small logarithms next to the large logarithms containing the common scale. However, in the case of contributions which depend on powers of the new physics scale the situation is completely different, and the presence of several masses could change completely the picture of unification. Cutoffs can give an indication of the presence of power corrections, but the coefficients of these corrections cannot be computed without knowing the details of the full theory.

To see this point more clearly, we add to our $4+\delta$ dimensional theory an additional fermion with mass $M_{f}$ satisfying $M_{s} \gg M_{f} \gg M_{c}$, such that compactification corrections may be neglected, and compute its effects on the coupling constant for $M_{c}^{2} \ll Q^{2} \ll M_{f}^{2}$, using dimensional regularization. We have

$$
\Pi_{f}^{(\delta)}(Q)=\frac{e_{4}^{2}}{2 \pi^{2}}\left(\frac{\pi}{M_{c}}\right)^{\delta / 2} \Gamma(-\delta / 2) \int_{0}^{1} d x x(1-x)\left(M_{f}^{2}+x(1-x) Q^{2}\right)^{\delta / 2}
$$

By expanding for $Q^{2} \ll M_{f}$ and integrating over $x$ we obtain

$$
\Pi_{f}^{(\delta)}(Q)=\frac{e_{4}^{2}}{2 \pi^{2}}\left(\sqrt{\pi} \frac{M_{f}}{M_{c}}\right)^{\delta} \Gamma\left(-\frac{\delta}{2}\right)\left(\frac{1}{6}+\frac{\delta}{60} \frac{Q^{2}}{M_{f}^{2}}\right) .
$$

For odd values of $\delta$ we can use the analytic continuation of the $\Gamma$ function to obtain a finite result. For even values of $\delta$ we will allow a slight departure of the integer value in order to dimensionally regularize the integral. Clearly, integrating out the heavy fermion gives power corrections to the gauge coupling. In addition, it also generates contributions to the higher dimension operators, e.g. contributions proportional $Q^{2}$ and higher powers. As can be seen 
by comparing with Eqs. (4.6)-(4.9) these power corrections are qualitatively similar to those calculated using a hard cutoff. Evidently, in the context of a more complete theory (in this case, given the existence of a heavy fermion), power corrections may be encountered even if the dimensional regularization is employed. However, as one can easily see by setting, for example, $\delta=1$ in Eq.(5.8), the coefficients of the power corrections obtained knowing the full theory are in general different from those obtained using a hard cutoff, e.g. Eq.(4.7). In fact, no choice of $\Lambda$ in Eq.(4.7) can reproduce all the coefficients appearing in Eq.(5.8).

The situation is somewhat similar to what happens when $\chi P T$ with $S U(2) \otimes S U(2)$ is matched to $\chi P T$ with $S U(3) \otimes S U(3)$. In the $S U(2) \otimes S U(2)$ theory, just by dimensional arguments, one can expect corrections like $m_{K}^{2} / f_{\pi}^{2}$. But, can one compute them reliably without even knowing that there are kaons?

\section{CONCLUSIONS}

We have attempted a critical discussion of the arguments in favor of power-law running of coupling constants in models with extra dimensions. We have shown that the naive arguments lead to an arbitrary $\beta$ function depending on the particular way chosen to cross KK thresholds. In particular, if one chooses the physical way of passing thresholds provided by the vacuum polarization function of the photon, a $\beta$ function that counts the number of modes is divergent for more than 5 dimensions.

We have studied the question of decoupling of KK modes in QED with $4+\delta$ (compact) dimensions by analyzing the behavior of the VPF of the photon. We have computed first the imaginary part of the VPF by using unitarity arguments, and found that it rapidly reaches the value obtained in a non-compact theory (only a few modes are necessary). We also showed that it grows as $\left(s / M_{c}^{2}\right)^{\delta / 2}$, exhibiting clearly the non-renormalizability of theories in extra dimensions. To obtain the full VPF, one can use an appropriately subtracted dispersion relation. Instead, we use the full quantum effective field theory, with the expectation, suggested by the calculation of the imaginary part of the VPF, that the bad UV behavior of the theory is captured by the behavior of the uncompactified theory. To check this idea, we have computed the VPF in the uncompactified theory, regularized by dimensional regularization $(\delta \rightarrow \delta-\varepsilon)$. We have found that, after analytical continuation, the one loop VPF is finite, and proportional to $Q^{\delta}$ for odd number of dimensions, and 
has a simple pole, proportional to $Q^{\delta}$, for even number of dimensions. This result can be understood easily, because there are no possible Lorentz and gauge invariant operators in the Lagrangian able to absorb a term like $Q^{\delta}$ for odd $\delta$. For $\delta$ even it shows that higher dimension operators are needed to regularize the theory. As a check we also recovered the imaginary part of the VPF in the limit of infinite compactification radius.

For comparison with other approaches, we have also obtained the VPF in the case that a hard cutoff is used to regularize it. We found that the pieces that do not depend on the cutoff are exactly the same as those obtained by dimensional regularization, while the cutoff dependent pieces are arbitrary, and can be changed at will by changing the cutoff procedure.

Next we have computed the VPF in the compactified theory, and showed that it can be separated into a UV and IR finite contribution and the VPF calculated in the uncompactified theory; as was shown previously, the latter can be controlled using dimensional regularization. The finite part is more complicated, but can be computed numerically for any number of dimensions. Also, some analytical approximations have been obtained for the low and the high energy limits $\left(Q \ll M_{c}\right.$ and $Q \gg M_{c}$ respectively). Adding these two pieces, together with the counterterms coming from higher dimension operators, we obtain a finite expression for an effective charge which can be extrapolated continuously from $Q \ll M_{c}$ to $Q \gg M_{c}$; however, its value does depend on higher dimension operator couplings.

Decoupling of all KK modes in this effective charge is smooth and physically meaningful, and the low energy logarithmic running is recovered. We use this effective charge to connect the low energy couplings (i.e. $\alpha_{\text {eff }}\left(m_{Z}\right)$ ) with the coupling of the theory including all KK modes, regularized by dimensional regularization. We find that this matching only involves the standard logarithmic running from $m_{Z}$ to the compactification scale $M_{c}$. In particular, no power corrections appear in this matching. However, if cutoffs are used to regularize the VPF in the non-compact space, one does find power corrections, exactly as expected from naive dimensional analysis. In the EFT language one could interpret these corrections as an additional matching between the effective $D$ dimensional field theory and some more complete theory. The question is how reliably can this matching be estimated without knowing the complete theory. By adding to our theory an additional fermion with $M_{f} \gg M_{c}$, and integrating it out, we argue that power corrections cannot be computed without knowing the details of the complete theory, in which the $D$ dimensional theory is embedded. Some 
examples in which this matching can, in principle, be computed are some string models [8, 14, 57], and the recently proposed de-constructed extra dimensions [58, 59, 60]. For the question of unification of couplings this result seems rather negative, at least when compared with standard Grand Unified Theories, where gauge coupling unification can be tested without knowing their details. Alternatively, one can approach this result from a more optimistic point of view, and regard the requirement of low-energy unification of couplings as a stringent constraint on the possible completions of the extra-dimensional theories [18, 61].

\section{Acknowledgments}

This work has been funded by the Spanish MCyT under the Grants BFM2002-00568 and FPA2002-00612, and by the OCyT of the "Generalitat Valenciana" under the Grant GV01-94.

[1] N. Arkani-Hamed, S. Dimopoulos, and G. R. Dvali, Phys. Rev. D59, 086004 (1999), hep-ph/9807344.

[2] N. Arkani-Hamed, S. Dimopoulos, and G. R. Dvali, Phys. Lett. B429, 263 (1998), hep-ph/9803315.

[3] I. Antoniadis, Phys. Lett. B246, 377 (1990).

[4] I. Antoniadis and K. Benakli, Phys. Lett. B326, 69 (1994), hep-th/9310151.

[5] C. P. Bachas, JHEP 11, 023 (1998), hep-ph/9807415.

[6] K. R. Dienes, E. Dudas, and T. Gherghetta, Phys. Lett. B436, 55 (1998), hep-ph/9803466.

[7] K. R. Dienes, E. Dudas, and T. Gherghetta, Nucl. Phys. B537, 47 (1999), hep-ph/9806292

[8] D. Ghilencea and G. G. Ross, Phys. Lett. B442, 165 (1998), hep-ph/9809217.

[9] C. D. Carone, Phys. Lett. B454, 70 (1999), hep-ph/9902407.

[10] A. Delgado and M. Quiros, Nucl. Phys. B559, 235 (1999), hep-ph/9903400.

[11] E. G. Floratos and G. K. Leontaris, Phys. Lett. B465, 95 (1999), hep-ph/9906238.

[12] P. H. Frampton and A. Rasin, Phys. Lett. B460, 313 (1999), hep-ph/9903479.

[13] Z. Kakushadze, Nucl. Phys. B548, 205 (1999), hep-th/9811193.

[14] Z. Kakushadze and T. R. Taylor, Nucl. Phys. B562, 78 (1999), hep-th/9905137. 
[15] A. Perez-Lorenzana and R. N. Mohapatra, Nucl. Phys. B559, 255 (1999), hep-ph/9904504.

[16] D. Dumitru and S. Nandi, Phys. Rev. D62, 046006 (2000), hep-ph/9906514.

[17] M. Masip, Phys. Rev. D62, 065011 (2000), hep-ph/0001115.

[18] N. Arkani-Hamed, A. G. Cohen, and H. Georgi, Accelerated unification, 2001, hep-th/0108089.

[19] Z. Berezhiani, I. Gogoladze, and A. Kobakhidze, Phys. Lett. B522, 107 (2001), hep-ph/0104288.

[20] A. Hebecker and A. Westphal, Power-like threshold corrections to gauge unification in extra dimensions, 2002, hep-ph/0212175.

[21] J. Kubo, H. Terao, and G. Zoupanos, Nucl. Phys. B574, 495 (2000), hep-ph/9910277.

[22] R. Contino, L. Pilo, R. Rattazzi, and E. Trincherini, Nucl. Phys. B622, 227 (2002), hep-ph/0108102.

[23] G. Rodrigo and A. Santamaria, Phys. Lett. B313, 441 (1993), hep-ph/9305305

[24] H. Georgi, Ann. Rev. Nucl. Part. Sci. 43, 209 (1993).

[25] K. G. Wilson and J. B. Kogut, Phys. Rept. 12, 75 (1974).

[26] S. Weinberg, Physica A96, 327 (1979).

[27] S. Weinberg, Phys. Lett. B91, 51 (1980).

[28] L. J. Hall, Nucl. Phys. B178, 75 (1981).

[29] H. Leutwyler, Ann. Phys. 235, 165 (1994), hep-ph/9311274.

[30] A. V. Manohar, Effective field theories, 1996, hep-ph/9606222.

[31] A. Pich, Effective field theory, 1998, hep-ph/9806303.

[32] E. Witten, Nucl. Phys. B122, 109 (1977).

[33] H. Georgi, H. R. Quinn, and S. Weinberg, Phys. Rev. Lett. 33, 451 (1974).

[34] J. Callan, Curtis G., S. R. Coleman, J. Wess, and B. Zumino, Phys. Rev. 177, 2247 (1969).

[35] S. R. Coleman, J. Wess, and B. Zumino, Phys. Rev. 177, 2239 (1969).

[36] S. Weinberg, Phys. Rev. 166, 1568 (1968).

[37] J. Gasser and H. Leutwyler, Ann. Phys. 158, 142 (1984).

[38] A. Pich, Rept. Prog. Phys. 58, 563 (1995), hep-ph/9502366.

[39] G. Ecker, Prog. Part. Nucl. Phys. 35, 1 (1995), hep-ph/9501357.

[40] C. P. Burgess and D. London, Phys. Rev. Lett. 69, 3428 (1992).

[41] C. P. Burgess and D. London, Phys. Rev. D48, 4337 (1993), hep-ph/9203216.

[42] M. Quiros, J. Phys. G27, 2497 (2001). 
[43] S. Hamidi and C. Vafa, Nucl. Phys. B279, 465 (1987).

[44] L. J. Dixon, D. Friedan, E. J. Martinec, and S. H. Shenker, Nucl. Phys. B282, 13 (1987).

[45] D. Binosi and J. Papavassiliou, The qcd effective charge to all orders, 2002, hep-ph/0209016.

[46] W. Bernreuther and W. Wetzel, Nucl. Phys. B197, 228 (1982).

[47] G. Rodrigo, A. Pich, and A. Santamaria, Phys. Lett. B424, 367 (1998), hep-ph/9707474.

[48] S. J. Brodsky, M. S. Gill, M. Melles, and J. Rathsman, Phys. Rev. D58, 116006 (1998), hep-ph/9801330.

[49] T. Appelquist, H.-C. Cheng, and B. A. Dobrescu, Phys. Rev. D64, 035002 (2001), hep-ph/0012100.

[50] A. Pomarol and M. Quiros, Phys. Lett. B438, 255 (1998), hep-ph/9806263.

[51] A. Delgado, A. Pomarol, and M. Quiros, JHEP 01, 030 (2000), hep-ph/9911252.

[52] J. Papavassiliou and A. Santamaria, Phys. Rev. D63, 125014 (2001), hep-ph/0102019

[53] A. Muck, A. Pilaftsis, and R. Ruckl, Phys. Rev. D65, 085037 (2002), hep-ph/0110391

[54] T. R. Taylor and G. Veneziano, Phys. Lett. B212, 147 (1988).

[55] C. Itzykson and J. B. Zuber, QUANTUM FIELD THEORY (Mcgraw-hill, New York, USA, 1980), 705 Pag.

[56] M. E. Peskin and D. V. Schroeder, An Introduction to quantum field theory (Addison-Wesley, Reading, USA, 1995), 842 pag.

[57] K.-w. Choi, H. D. Kim, and Y. W. Kim, JHEP 11, 033 (2002), hep-ph/0202257.

[58] N. Arkani-Hamed, A. G. Cohen, and H. Georgi, Phys. Rev. Lett. 86, 4757 (2001), hep-th/0104005.

[59] H.-C. Cheng, C. T. Hill, S. Pokorski, and J. Wang, Phys. Rev. D64, 065007 (2001), hep-th/0104179.

[60] S. Pokorski, J. Phys. G27, 2479 (2001), hep-th/0107052.

[61] P. H. Chankowski, A. Falkowski, and S. Pokorski, JHEP 08, 003 (2002), hep-ph/0109272. 